\title{
Prediction of Two-Element Cylindrical Electrostatic Lens Parameters Using Dynamic Artificial Neural Network
}

\author{
A.H. ISIK* \\ Department of Computer Engineering, Mehmet Akif Ersoy University, Burdur, Turkey \\ (Received August 13, 2014; in final form January 25, 2015)
}

\begin{abstract}
Two-element cylindrical electrostatic lens systems allowed to control low energy electron or charged particle beam have great importance. In this context, dynamic artificial neural network using nonlinear autoregressive exogenous model has been utilized to predict optimum linear magnification and overall voltage values for these lens designs. The focusing characteristics of electron beam in two-element cylindrical lens systems are investigated with two different nonlinear autoregressive exogenous based artificial neural network models. First artificial neural network model is employed for predicting of voltage ratios of lenses and magnification values. This model interpolates among the object $(P)$ and image positions $(Q)$ and finally finds optimum voltage ratios and magnification values through training dataset. Due to the deviations of electron trajectories in a real lens system, the spherical aberration effects are also taken into account to determine the optimal lens parameters. Therefore, the second artificial neural network model is constructed for predicting spherical aberration coefficients in image point. For each of artificial neural network models, training, test and validation data set are obtained from SIMION 8.1 ion and electron optics software. Artificial neural network model outputs are compared with the SIMION data and very good agreements are found. While artificial neural network is frequently applied in different fields, this is the first study that uses dynamic artificial neural network to predict the parameters of electrostatic lens. It is believed that this pioneering work will be a guide for the future investigations in lens design systems.
\end{abstract}

DOI: 10.12693/APhysPolA.127.1717

PACS: 84.35.+i, 07.05.Mh, 41.85.Ne

\section{Introduction}

Electrostatic lenses are useful optical tools in controlling beams of electrons or charged particles. Therefore, a development of electrostatic lenses has a great interest for a long time and has been focused on the determination of optical properties with high accuracy over the last years $[1,2]$. Among these lenses, two-element cylindrical electrostatic lens is widely used geometry in low energy charged particle selection systems [3-9]. Experiments of low energy electrons or charged particles require detailed research for two-element cylindrical electrostatic lens area. This investigation depends on the understanding of sensitive changes in optical parameters of lenses as a function of their voltage ratios. The influence of voltages can be directly observed in object-image position diagram ( $P-Q$ diagram) called also as design curves. In this diagram, the magnification and voltage ratios can both be precisely determined in given relation between the object $(P)$ and image positions $(Q)$ of charged particles. The only experimental $P-Q$ curves data is given by Spangenberg and Field for real object $(P)$ and image positions $(Q)[9]$. During the development of optical systems for charged particle beams, different methods have been employed to provide an optimum design in low-energy charged particle spectrometers [6-10]. Spherical aberrations coefficients for two-element lenses have been investigated using several methods [11-12].

*e-mail: ahakan@mehmetakif.edu.tr
In recent years, the artificial intelligent algorithms have become increasingly popular in many fields of physics that have a wide range of applications [13-15]. These new computer-aided algorithms are similar computational tool designed on the nervous system of human brain and provide successful results [16]. In this approximation, network learn the relation between input and desired output data with a set of given examples and discover an optimum output data for an unknown input. During the network training process, the information transmits from one neuron to another. Thus, information from example data set is processed for a suitable output data. Over the past decade, artificial intelligent applications have been developed to increase the performance of charged particle probe instruments [1721]. In this study, nonlinear autoregressive exogenous (NARX) model based artificial neural network (ANN) which is a kind of artificial intelligent algorithm has been used. The main objective is to demonstrate the benefit and sensitivity of NARX based ANN prediction for twoelement cylindrical electrostatic lens. In this approach, we can find future values of lens parameters depending on the training dataset.

This study is organized as follows. Section 2 of this study describes two-element electrostatic lens and lens parameters to control charged particle beams. Detailed information about the NARX based dynamic artificial neural network model is given in Sect. 3. The comparison results are given in Sect. 4. In this section, the MATLAB simulation results have been compared with SIMION software data. Finally, the conclusion and future works regarding the prediction of electrostatic lens parameters are given. 


\section{Charged particle lens systems}

The motion of charged particles in lens region should be calculated for maximum ease of use in experiments. The motion of charged particles can be described with the first and second focal points ( $F_{1}$ and $F_{2}$, respectively) and the object $(P)$ and image points $(Q)$. Two-element electrostatic lenses are commonly used to control electron beam especially in low energies. These lenses consist of two closely spaced cylinders held at potentials $V_{1}$ and $V_{2}$. They have been modeled and illustrated in Fig. 1. In this figure, $D$ represents the diameter of lens. $D$ is commonly used as a unit of length for all parameters such as $P / D$. The pattern of spherical aberration in image point is also represented in Fig. 1.

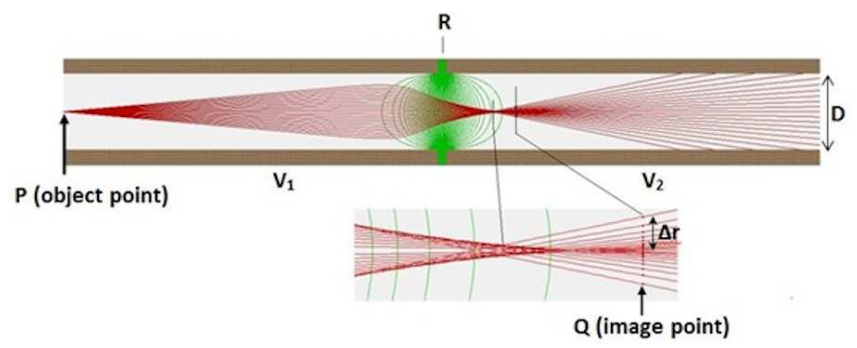

Fig. 1. Cross-sectional view of a two element cylindrical electrostatic lens. Equipotential lines are represented with green lines for the applied different voltages $\left(V_{1}\right.$ and $\left.V_{2}\right)$. The paths of charged particle beam are shown as blue rays for different trajectory.

The paths of charged particles are determined by fundamental lens parameters. One of the parameters is linear magnification $(M)$. Linear magnification is calculated from the ratio of the final to initial beam diameter, $r_{2} / r_{1}$, respectively. In addition, $M$ can be obtained by using

$$
M=-\frac{f_{1}}{P-F_{1}}=-\frac{Q-F_{2}}{f_{2}},
$$

where the first and second mid-focal points are represented by $f_{1}$ and $f_{2}$, respectively. In image point, the beam diameter is defined by the linear magnification. Another important parameter in lens design is spherical aberration coefficient in image point $(Q)$. Electrons entering the lens system from a different angle are focused in different image points. This is called as a spherical aberration effect in lenses. For given rays with different half angle $\left(\alpha_{0}\right)$ in object point, the spherical aberration in image point $(Q)$ is represented in Fig. 1. The spherical aberration coefficient $\left(C_{\mathrm{s}}\right)$ is defined by

$$
\Delta r=-M C_{\mathrm{s}} \alpha_{0}^{3},
$$

where $\Delta r$ is the radius of the disc in image plane, $M$ is the linear magnification, and $\alpha_{0}$ is the maximum half angle for electrons entering from a given object distance $(P)$. The ratio of $C_{\mathrm{s}} / D$ for decelerating lens $\left(V_{1} / V_{2}\right)$ to accelerating lens $\left(V_{2} / V_{1}\right)$ are obtained with the formula

$$
\frac{C_{\mathrm{s}}\left(V_{1} / V_{2}\right)}{C_{\mathrm{s}}\left(V_{2} / V_{1}\right)}=M^{4}\left(\frac{V_{2}}{V_{1}}\right)^{3 / 2},
$$

where $C_{\mathrm{S}}\left(V_{1} / V_{2}\right)$ stands for the spherical aberration coefficient when electron is retarded from second lens voltage $\left(V_{2}\right)$ to first lens voltage $\left(V_{1}\right)[7]$.

On the other hand, lens voltages are affected the trajectories of charged particle beam. Therefore, the potential $(V(r))$ has to be calculated to find the trajectory of a particle in lens region. There are different methods such as the boundary element method [7], finite difference method [8], and finite-element method [22] for the precise calculation of the potential distributions along the optical axis of charged particle beam. Among these methods, SIMION program uses the finite-difference method to solve the Laplace equation [23]. The Laplace equation is

$$
\nabla^{2} V_{(r, z)}=0
$$

where $V_{(r, z)}$ is the potential in lens region. When the potential at any point in the trajectory of a charged particle is calculated, the working space is changed for the calculation of potential in a neighbor point by iteration technique. The working space is described as a regular lattice of different neighbor points. Figure 2 represents

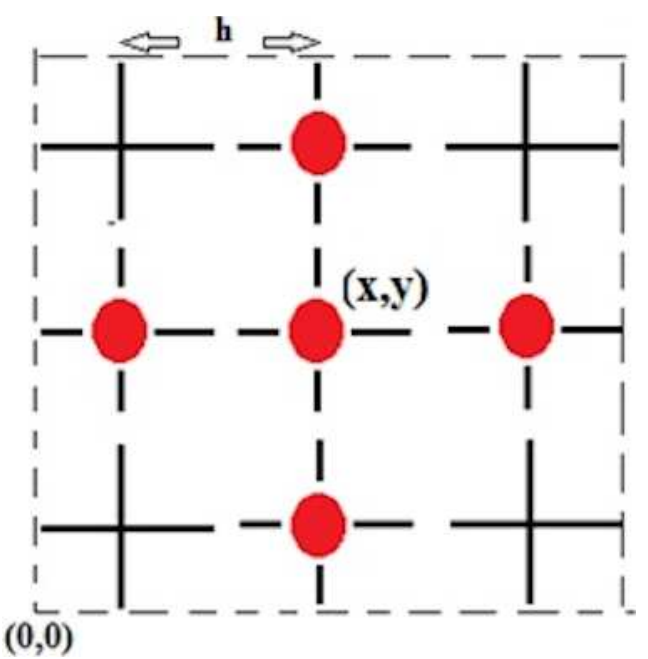

Fig. 2. A square lattice of points separated by lengths $h$. Points are marked by red circles. The center point is at $(\mathrm{x}, \mathrm{y})$.

the lattice of five points. In five-point finite difference method, the potential of center point in this lattice can be found with the aid of the four outer point potentials. In Cartesian coordinates, the potential at center point on the lattice is calculated by using Eq. (3) as shown below

$$
V_{(x, y)}=\frac{V_{(x-h, y)}+V_{(x, y-h)}+V_{(x+h, y)}+V_{(x, y+h)}}{4} .
$$

SIMION software solves the Laplace equation and calculates the paths of charged particles in specified workspace. This software uses nine-point finite difference method. In nine point difference method, four further neighbor points are calculated to increase the accuracy of the numerical solution. This calculation takes more time depending on the number of iteration. 


\section{Designing of NARX based dynamic artificial neural network model}

Prediction algorithms have become popular among the academic field. One of the main reasons is the capability of these algorithms in solving undefined relationship. The low computational burden is also another characteristic of these algorithms that provides desired output in a short minute. Some of the best known prediction algorithms are artificial neural network, genetic algorithm, particle swarm optimization [24, 25]. It has been proved that artificial neural network has better performance than some of algorithms in terms of nonlinearity, and limited data [26]. Therefore, this algorithm is preferred for data analysis. There are several types of artificial neural network model such as multilayer feed forward, selforganizing map, radial basis function and NARX. NARX is a kind of recurrent artificial neural network, which has feedback communication. In this type of $\mathrm{ANN}$, we can predict next values of a time series using previous values of time series data. It is commonly used in time-series problem and provides promising result for long-term dependences. NARX based ANN can solve complex relationship between input and output data. Additionally, fault tolerance makes this neural network far superior to the well-known prediction algorithm. Due to the distinguished features, NARX based dynamic artificial neural network model is used for prediction of two-element electrostatic lens parameters. These parameters are compatible with time series data format. Time series refer to the sequence of vector points, $y(t), t=0,1, \ldots$, where $t$ corresponds to passing time. Dynamic artificial neural network focuses on predicting of time series data at time $t+d$, from the $N$ time before at time $t$. The NARX equation is

$$
\begin{aligned}
& y(t+d)=f\left(x(t), x(t-1), x(t-2), \ldots, x\left(t-n_{x}\right),\right. \\
& \left.y(t-1), y(t-2), \ldots, y\left(t-n_{y}\right)\right),
\end{aligned}
$$

where $x(t)$ denotes input of the time series at time $t$, and $f_{i}$ is a nonlinear function. $d, n_{x}$ (input delay) and $n_{y}$ (output delay) are the delays of the system [27]. In this equation, $y(t+d)$ is the output of the time series and obtained from former values of input and output values. The estimation of future values of vector $y(i)$, based on the training data, allow addressing of time-critical conditions. The architecture of designed NARX based ANN model is shown in Fig. 3 [29]. This NARX model consists of input, hidden, and output layers. The object $(P)$ and image positions $(Q)$ are used as input variables. There is no common rule regarding the number

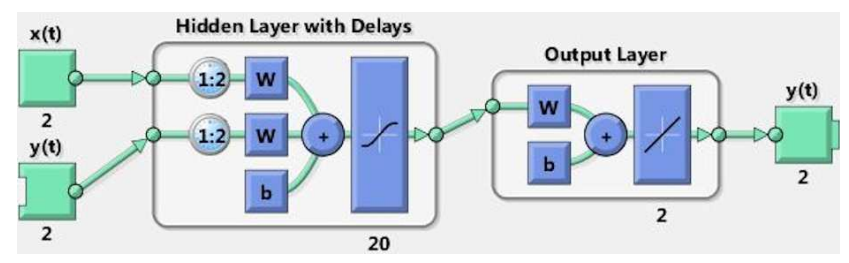

Fig. 3. Architecture of NARX based ANN model. of hidden layers. The number is generally found with trial and error method [29]. In designed model, there are twenty neurons in hidden layer and sigmoid transfer function is utilized. In addition, feedback delayed outputs values of $y(t)$ are used as input variable. In this way, the best prediction of voltage ratios and magnification values is achieved.

\section{Results}

In the first step of the artificial neural network model development, two-element electrostatic lens system is simulated using the SIMION 3D 8.1 ion and electron optics software. Obtained SIMION data is used as training, test and validation dataset for ANN model. Based on time series approach, appropriate dynamic ANN model is constructed to predict the voltage ratios and magnification value for two-element electrostatic lens design. Designed time-series ANN model consists of two input neurons ( $P$ and $Q$ ) and two output neurons ( $M$ and $V_{2} / V_{1}$ ). Data analysis is carried out with 100 sample data. MATLAB Neural Network Toolbox and Statistics Toolbox is utilized. In MATLAB analysis, ANN model is trained with $80 \%$ of SIMION data. In this way, bias and weights are adjusted. Testing and validation are carried out through $5 \%$ and $15 \%$ of SIMION data, respectively. The SIMION input data is first normalized with the following formula to the range of -1 to +1 to achieve zero mean. Normalization formula is given with

$$
X_{\text {normalized }}=\frac{X_{i}-X_{\min }}{X_{\max }-X_{\min }},
$$

where $X_{i}$ represents the values to be normalized and the indices of min and max denote the minimum and maximum values for a set data, respectively. The prediction performance of the ANN model has been evaluated with four statistical metrics. These are mean square error (MSE), error histogram, regression, and input-error cross-correlation.

Mean square error (MSE) is determined as graphical demonstration of the scattered data. Error histogram is the average of the squared distinction between ANN predicted values $\left(X_{p t}\right)$ versus SIMION output values $\left(X_{t}\right)$ for a number of $N$ trials [30]. MSE is found with

$$
\mathrm{MSE}=\frac{1}{N} \sum_{t=1}^{N}\left(X_{p t}-X_{t}\right)^{2} .
$$

Error histogram of designed network is presented in Fig. 4a. This histogram shows the distribution of errors with the training, test, and the validation dataset. In this histogram, zero error means concentrated point of error which is shown by orange line. The mean square errors at each epoch for the three different datasets are shown in Fig. 4b. The MSE value of the designed model was $3.129 \times 10^{-7}$ and was achieved at the 264 th epoch. In this analysis, the process of training stops when the validation error reaches steady-state. The green line shows the MSE for the validation dataset, the blue line for the training set, the red line demonstrate for the test set. These results indicate that there is a high performance result. 


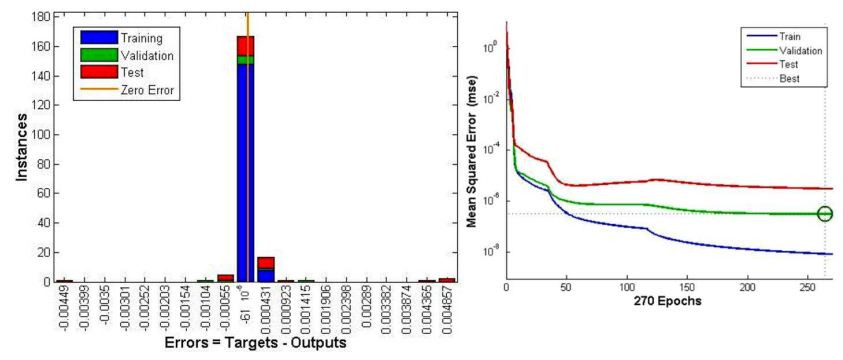

Fig. 4. (a) Error histogram of a 20 hidden layered time series neural network indicate zero mean Gaussian distribution. (b) Mean square error of training, validation and test datasets.

The cross correlation sequence values versus lag (delay) number are shown in Fig. 5. The correlation between errors and the input sequence $x(t)$ is found with input-error cross-correlation. For absolutely accurate estimation, whole correlations must be zero. It is seen in this Fig. 5 that there is an accurate cross-correlation.

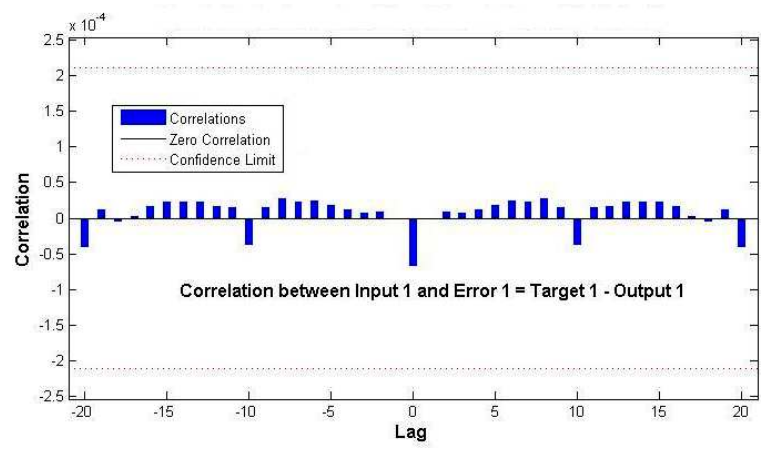

Fig. 5. Cross-correlation of designed ANN model.
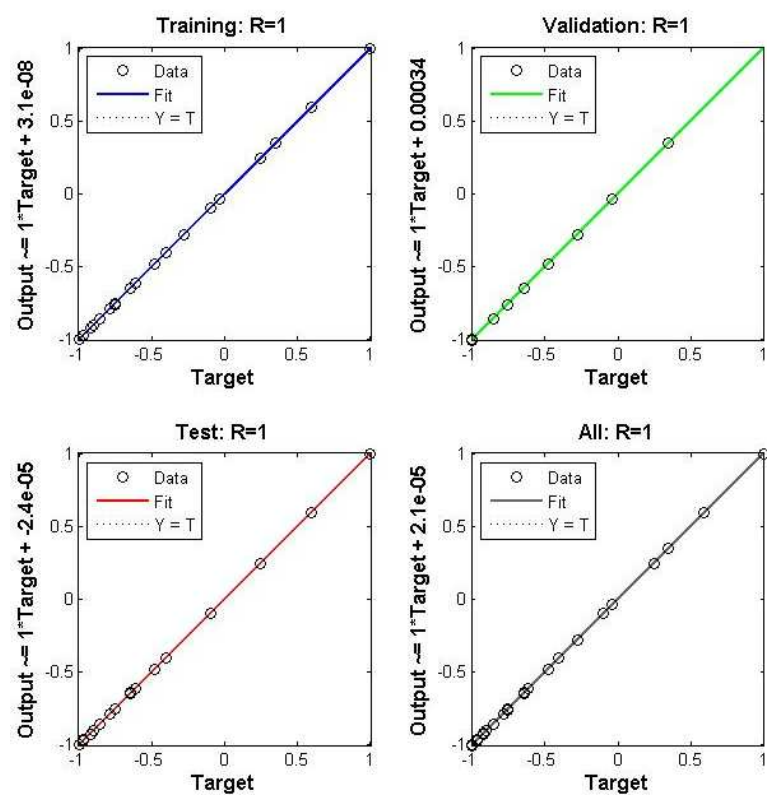

Fig. 6. Regression $(R)$ values of training, validation and test datasets.
The correlation between outputs and target values are determined through regression $(R)$ value. For a detailed analysis of ANN model, regression results of training, validation and test dataset are presented in Fig. 6. It was found that ANN model has the high regression values in training, validation, and testing are 1,1 , and 1 , respectively. An $R$ value of 1 indicates a close relationship, 0 a weak relationship. Linear regression equation is given with the formula of $y=a x+b$, where $b$ is the crossing point of $y$ and $a$ is the slope of the graph given below

$$
\begin{aligned}
a & =\frac{\sum y-b \sum x}{n}, \\
b & =\frac{n \sum(x y)-\left(\sum x\right)\left(\sum y\right)}{n \sum x^{2}-\left(\sum x\right)^{2}},
\end{aligned}
$$

where $x$ is the input variable and $n$ is the time period.

Comparison of the normalized ANN output data with the SIMION data is given in Fig. 7a. To provide a visual reference, the comparison between the predicted ANN data and SIMION data including the error bars is presented in Fig. 7b. The comparison results show that the ANN prediction accuracy of voltage ratios for twoelement electrostatic cylinder lenses close to $97 \%$.

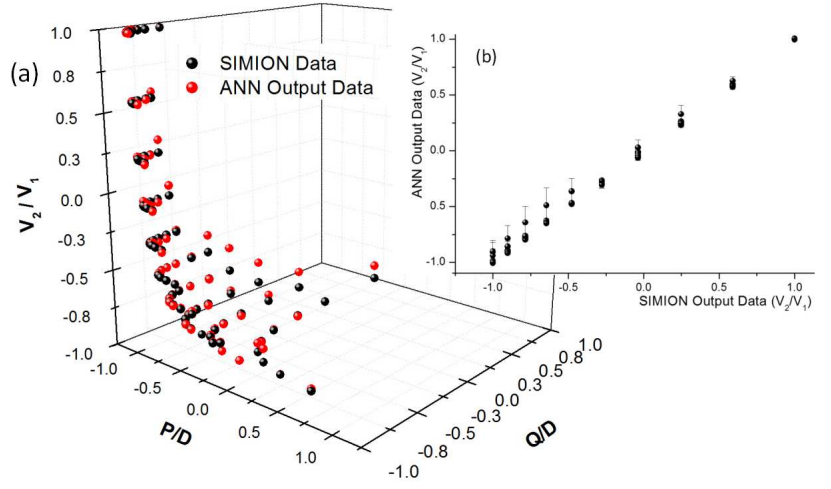

Fig. 7. (a) Predicted data of ANN model for a set of voltage ratios of two element lens with $P$ and $Q$ values against SIMION input data set. (b) The comparison of SIMION and ANN model prediction data.

Another time series ANN model is constructed to obtain optimum situation of lens design. In this approach, spherical aberration coefficients in image points $(Q / D)$ are predicted using $M$ and voltage ratios for the same lens design. The Levenberg-Marquardt algorithm is used to train ANN. Regression analysis is carried out to measure the performance of this ANN model. Analysis results are given in Fig. 8. ANN model has $98.8 \%$ for training, $99 \%$ for test, $98.2 \%$ for validation regression values, respectively. The obtained results show that time series ANN model can be used to predict spherical aberration coefficients using voltage ratios and magnification values in cylindrical electrostatic lens. This analysis is a very simple technique for predicting the aberrations with high accuracy. 

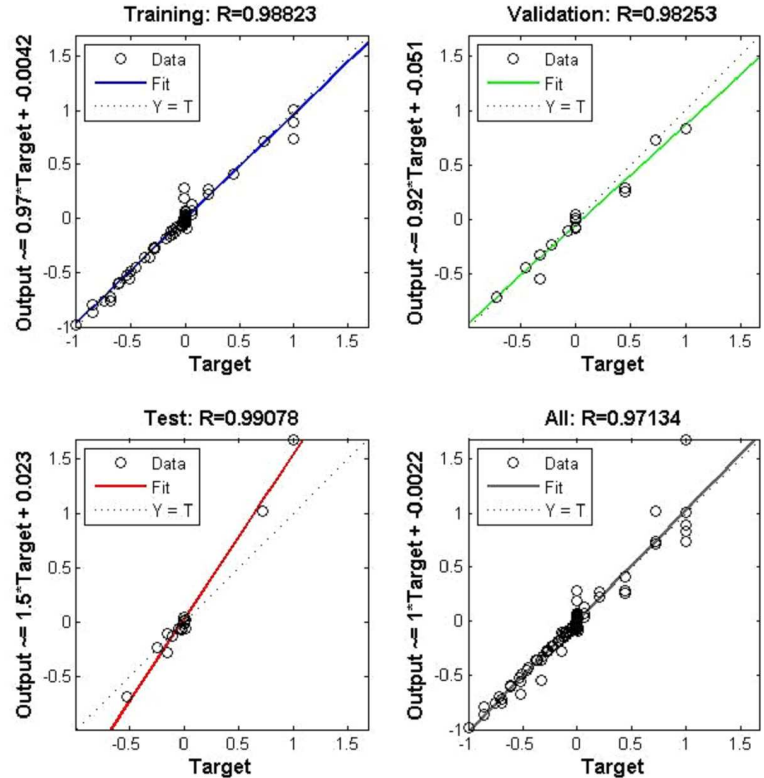

Fig. 8. Regression $(R)$ values of $\mathrm{ANN}$ for obtaining spherical aberrations.

\section{Conclusions}

In this study, NARX based dynamic artificial neural network is used to predict optical properties of twoelement cylindrical electrostatic lens. To my knowledge, this is the first study that uses dynamic ANN to predict the focusing parameters of electron beam with low spherical aberrations in image point. It is clearly shown that the results obtained from ANN are in good agreement with the reference data. In this approach, future values of lens parameters can also be found with high sensitivity. Instead of simulation data, this technique can be validated with experimental data. In addition, different types of heuristic algorithms can be applied to the electrostatic lens systems to predict parameters in the future.

\section{Acknowledgments}

I would like to thank Nimet Isik for her valuable information concerning the electrostatic lens and SIMION researchers for delivering a test data for checking our results.

\section{References}

[1] O. Sise, M. Ulu, M. Dogan, Nucl. Instrum. Methods Phys. Res. A 554, 114 (2005).

[2] O. Sise, N. Okumus, M. Ulu, M. Dogan, J. Electron Spectrosc. Relat. Phenom. 175, 76 (2009).

[3] G.E. Chamberlain, Phys. Rev. 155, 46 (1967).
[4] C.J. Kuyatt, J.A. Simpson, Rev. Sci. Instrum. 38 103 (1967).

[5] F.H. Read, J. Phys. E 2, 165 (1969).

[6] F.H. Read, J. Comer, R.E. Imhof, J.N.H. Brunt, E. Harting, J. Electron. Spectrosc. Relat. Phenom. 4, 293 (1974)

[7] F.H. Read, A. Adams, J.R. Soto-Montie, J. Phys. E 4, 625 (1971).

[8] S. Natali, D.D. Chio, C.E.J. Kuyatt, Res. Natl. Bur Stand. Sec. A 76A, 27 (1972).

[9] K. Spangenberg, L. Field, IEEE Conf. Proc. 30, 138 (1942).

[10] D.W.O. Heddle, J. Phys. D Appl. Phys. 32, 1447 (1999).

[11] T. Saito, O.J. Sovers, J. Appl. Phys. 50, 3050 (1979).

[12] O. Sise, M. Ulu, M. Dogan, Nucl. Instrum. Methods Phys. Res. A 573, 329 (2007).

[13] M.E. Sigman, S.S. Rives, J. Chem. Inf. Comput. Sci. 34, 617 (1994).

[14] J. Khan, J.S. Wei, M. Ringner, L.H. Saal, M. Ladanyi, F. Westermann, F. Berthold, M. Schwab, C.R. Antonescu, C. Peterson, P.S. Meltzer, Nat. Med. 7, 673 (2001).

[15] C. Caetano, J.L. Reis, J. Amorim, M. Lemes, A.D. Pino, Int. J. Quant. Chem. 111, 2732 (2011).

[16] S. Haykin, Neural Networks and Learning Machines, 3rd ed., Pearson Education, New Jersey 2009.

[17] E. Munro, J. Vac. Sci. Technol. B 8, 1657 (1990).

[18] M. Szilagyi, IEEE Conf. Proc. 73, 412 (1985).

[19] T. P. Cavalcanti, J. Instrum. 8, C04003 (2013).

[20] F.A. Ali, Int. J. Adv. Comp. Techn. 2, 66 (2010).

[21] H. J. Yang, B. P. Roe, J. Zhu, Nucl. Instr. Meth Phys. Res. A 574, 342 (2007).

[22] E. Munro, in: Conf. Proc. on Electron Microscopy, Ed. P. Favard, : Société français de microscopie electronique, Paris 1970 , p. 55

[23] D.W.O. Heddle, Electrostatic Lens Systems, 2nd ed., IOP Press, London 2000.

[24] H. Kolanoski, Nucl. Instr. Meth. Phys. Res. A 367, 14 (1995).

[25] R. Camattari, V. Guidi Astron. Astrophys. 570 , A17 (2014).

[26] G. Zhang, B. Eddy Patuwo, M.Y. Hu, Int. J. Forecast. 14, 35 (1998).

[27] R.J. Frank, N. Davey, S.P. Hunt, J. Intell. Robot Syst. 31, 91 (2001).

[28] M.V. Kumar, P. Sampath, S. Suresh, S.N. Omkar, R. Ganguli, Aircraft Eng. Aerosp. Techn. 83, 283 (2011).

[29] S.H. Arbain, A.J. Wibowo, Comput. Sci. 8, 1506 (2012).

[30] N. Sipöcz, F.A. Tobiesen, M. Assadi, Appl. Energy 88, 2368 (2011). 